\title{
Relationship Between Crystallization, Mechanical and Gas Barrier Properties of Poly(ethylene furanoate) (PEF) in Multinanolayered PLA-PEF and PET-PEF Films
}

Alain Guinault, Tiphaine Messin, Gilbert Anderer, Stefan Krawielitzki, Cyrille Sollogoub, Sebastien Roland, Anne Grandmontagne, Patrice Dole, Jean-Mario Julien, Catherine Loriot and Thierry Vincelot

Alain Guinault. PIMM, Arts et Metiers Institute of Technology, CNRS, Cnam, HESAM Universite, 151 Bd. de l'Hopital, 75013 Paris, France

Corresponding author: GUINAULT Alain, E-mail address: alain.guinault@lecnam.net

Tiphaine Messin. PIMM, Arts et Metiers Institute of Technology, CNRS, Cnam, HESAM Universite, 151 Bd. de l'Hopital, 75013

Paris, France

Gilbert Anderer. AVA Biochem, Zug, Switzerland

Stefan Krawielitzki. AVA Biochem, Zug, Switzerland

Cyrille Sollogoub. PIMM, Arts et Metiers Institute of Technology, CNRS, Cnam, HESAM Universite, 151 Bd. de l'Hopital, 75013

Paris, France

Sebastien Roland. PIMM, Arts et Metiers Institute of Technology, CNRS, Cnam, HESAM Universite, 151 Bd. de l'Hopital, 75013

Paris, France

Anne Grandmontagne. PIMM, Arts et Metiers Institute of Technology, CNRS, Cnam, HESAM Universite, 151 Bd. de l'Hopital, 75013 Paris, France

Patrice Dole. CTCPA, Bourg en Bresse, France

Jean-Mario Julien. Laboratoire national de métrologie et d'essais, Trappes, France

Catherine Loriot. Laboratoire national de métrologie et d'essais, Trappes, France

Thierry Vincelot. Laboratoire national de métrologie et d'essais, Trappes, France

Abstract. Food packaging films must be reinvented in order to answer the new demanding ecological requirements. Biobased and/or biodegradable polymers appear as an interesting alternative to reduce petroleum dependence and carbon dioxide emissions. Poly(ethylene furanoate) (PEF) appears today as a new promising biopolymer thanks to its good gas barrier and mechanical properties, despite its high price that could limit its industrial applications. Its combination with other polymers is thus of great interest and for the first time, film coextrusion process is used to create PLA-PEF and PET-PEF multi-micro/nano layered films. A new PEF grade developed by AVA Biochem in the H2020 Mypack program, has been used and firstly analysed in terms of melt processability, mechanical, thermal and gas barrier properties. Our major results confirmed the good gas barrier as well as mechanical properties of amorphous PEF. Post-extrusion PEF bulk thermal crystallization led to very brittle material making gas barrier measurements impossible. Micro/nanolayered PLA-PEF and PET-PEF films with different PEF layer thicknesses have been processed and post-extrusion annealing treatment was carried out. The relationship between crystallinity, mechanical and gas barrier properties will be investigated.

Keywords. Poly(Ethylene Furanoate), Multilayer, Multinanolayer, Mechanical Properties, Gas Barrier Properties, Nano Confinement

\section{Introduction}

Food packaging films must be reinvented in order to meet the new demanding requirements concerning the reduction of plastics consumption and petroleum dependence, and the limitation of the number of plastics used in order to ensure their recyclability. Using biobased polymers has been an explored strategy to meet the new environmental concerns.

Amongst the potential promising bioplastics, Poly(Lactic acid) (PLA) has been widely studied but presents some 
Relationship Between Crystallization, Mechanical and Gas Barrier Properties of Poly(eth...

drawbacks that limit its use, such as brittleness, low temperature stability and poor gas barrier properties [1]. It is therefore important to improve PLA gas barrier properties in order to reach the performance of poly(ethylene terephthalate) (PET) (around ten times better than PLA). Optimizing PLA crystallization may improve its gas barrier properties [2,3] and its thermal stability but leads generally to a dramatic loss of ductility. Poly(ethylene furanoate) (PEF) is another new promising biopolymer offering reduced barrier properties compared to PET (around 6 to 10 times) and similar temperature stability [4]. Still, two major drawbacks hinder its wide application: a high price and a high brittleness compared to PET. Among the various solutions to fill in those drawbacks, research efforts have been dedicated to combine PEF with another polymer, mostly PET, through copolymerization $[5,6]$ or blending $[7,8]$.

In this work, we combine PEF with PET or PLA in multilayer films for the first time, using coextrusion process. The addition of layer multiplying elements allows increasing the number of layers and induced orientation and confinement effects are expected $[9,10]$. The relationship between PEF crystallinity and thermal, mechanical and gas barrier properties will be highlighted.

\section{Material and methods}

\subsection{Materials}

PLA NPHT101, supplied by Natureplast, is a special formulation developed to promote crystallization (addition of nucleating agents) and to achieve high ductility (addition of a plasticizer). PET Indorama 101, supplied by Wipak, is an amorphous grade able to crystallize under biaxial stretching or thermal annealing. PEF was supplied by AVA Biochem under pellets form pretreated by a SSP (Solid State Polymerization) step process to reach high viscosity. The data of the AVA Biochem PEF grade are summarized in the table 1.

Table 1. Typical characteristics of the Ava Biochem PEF grade.

\begin{tabular}{|c|c|}
\hline Property & Value \\
\hline Intrinsic viscosity & $0.698 \mathrm{dl} / \mathrm{g}$ \\
\hline Relative viscosity & 1.950 \\
\hline Water content & $<0,008 \%$ \\
\hline $\mathrm{T}_{\mathrm{g}}$ & $92,3^{\circ} \mathrm{C}$ \\
\hline $\mathrm{T}_{\mathrm{m}}$ & $211.5^{\circ} \mathrm{C}$ \\
\hline $\mathrm{M}_{\mathrm{W}}$ & $54000 \mathrm{~g} / \mathrm{mol}$ \\
\hline $\mathrm{M}_{\mathrm{a}}$ & $21000 \mathrm{~g} / \mathrm{mol}$ \\
\hline Polydispersity Index & 2.57 \\
\hline Colour values & $\mathrm{L}: 64.68 ; \mathrm{a}:-4.52 ; \mathrm{b}:+20.21$ \\
\hline
\end{tabular}

\subsection{Methods}

\subsubsection{Processing}

\subsubsection{Layer multiplying co-extrusion}

PET-PEF and PLA-PEF films composed of 3, 128 or 512 alternating layers were processed using the layer multiplying device described elsewhere [11].The initial 3-layer feedblock was connected to two single-screw extruders (Scamex 20-20D) and 0, 6 and 8 multiplying elements were added to obtain respectively the 3-layers, the 128-layer and the 512-layer films. The amount of each polymer of the films was regulated thanks to gear pumps and by adjusting the 
screw speeds of each extruder to control the throughput. All the output films obtained were extruded through 2 different flat dies of $2 \mathrm{~mm}$ thickness and $150 \mathrm{~mm}$ in width for PET-PEF films and 200mm width for PLA-PEF films. The films were then stretched and cooled with chill rolls rotating at $2 \mathrm{~m} / \mathrm{min}$ for PET-PEF films and $1.2 \mathrm{~m} / \mathrm{min}$ for PLA-PEF films in order to produce $100 \mu \mathrm{m}$ thick films with the same draw ratio evaluated at around 20 for all the both PET-PEF and PLA-PEF films. Cooling temperatures were set at $70^{\circ} \mathrm{C}\left(40^{\circ} \mathrm{C}\right)$ for PET-PEF (PLA-PEF) films.

\subsubsection{Annealing after extrusion}

Extruded films were sandwiched between two Teflon sheets and were post-annealed using a SPECAC thermal press. Two different heating treatments were used depending on the films: PET-PEF films were annealed at $140^{\circ} \mathrm{C}$ for 2 hours while PLA-PEF were annealed in two steps, a first one at $80^{\circ} \mathrm{C}$ for 1 minute to induce PLA crystallization then a second at $140^{\circ} \mathrm{C}$ for 2 hours to crystallize PEF.

\subsubsection{Optical and electronic microscopy}

Films were embedded in a resin and the cross-section surface was polished. The cross-section surfaces were observed using Optical Microscopy (OM) in reflexion mode (all the films) and Scanning Electronic Microscopy (Philips SEM) (for 128 and 512-layer films).

\subsubsection{Thermal analysis}

Thermal analysis was carried out using a Perkin Elmer DSC 4000, under a nitrogen flow. Several milligrams of the films were used and heated from $30^{\circ} \mathrm{C}$ to $250^{\circ} \mathrm{C}$ at $10^{\circ} \mathrm{C} / \mathrm{min}$. The degree of crystallinity was calculated using the following values of the standard melting enthalpies $\left(\Delta \mathrm{H}_{0}\right): 187 \mathrm{~J} / \mathrm{g}$ for PEF [12], $93 \mathrm{~J} / \mathrm{g}$ for PLA and $140 \mathrm{~J} / \mathrm{g}$ for PET.

\subsubsection{Oxygen permeability measurements}

Oxygen permeability measurements were performed with a M/L 2/21 Mocon device according to ASTM D3985. The measurements were done at $23^{\circ} \mathrm{C}$ and $0 \% \mathrm{RH}$ and were duplicated. Only oxygen Transmission Rates (OTR) were reported since all the films had the same thicknesses $(100 \mu \mathrm{m})$.

\subsubsection{Mechanical properties}

Two kinds of mechanical tests were performed by LNE (Trappes, France): usual tensile tests and puncture tests for films. All the tests were performed at $23^{\circ} \mathrm{C}$ and $50 \% \mathrm{RH}$. Tensile tests were performed in the extrusion and transverse directions according to ISO 527-1. Measurements were systematically carried out on five specimens for each sample and modulus, strength and strains at yield and at break were deduced from the stress-strain curves. Moduli were measured at 2 to $5 \mathrm{~mm} / \mathrm{min}$ while the other characteristics were measured at $50 \mathrm{~mm} / \mathrm{min}$.

Puncture tests were carried out at $50 \mathrm{~mm} / \mathrm{min}$ with a Zwick/Roell device, according to NF H00-310 for the conical punch. The force and the energy for breaking were measured on 5 specimens for each sample.

\section{Results and discussion}

Reference films of neat PLA, PET and PEF films were first elaborated and the extrusion conditions for neat polymers were optimized (temperature and flat die). Then, the conditions of the co-extrusion process with layer multiplying elements were optimized. 
Relationship Between Crystallization, Mechanical and Gas Barrier Properties of Poly(eth...

Since PET must be extruded at temperature higher than $250^{\circ} \mathrm{C}$ in order to avoid crystallization at the die exit. and despite the fact that PEF must be usually extruded at temperatures between 220 and $240^{\circ} \mathrm{C}$ to avoid degradation, the layer multiplication device temperatures were set respectively to $250^{\circ} \mathrm{C}$ for PET-PEF films and $220^{\circ} \mathrm{C}$ for PLA-PEF films, in order to limit PLA degradation. $100 \mu \mathrm{m}$ thick films of neat polymers and multi-layered films with 10 to $15 \% \mathrm{w} / \mathrm{w}$ PEF amount were produced with 3, 128 and 512 alternating layers.

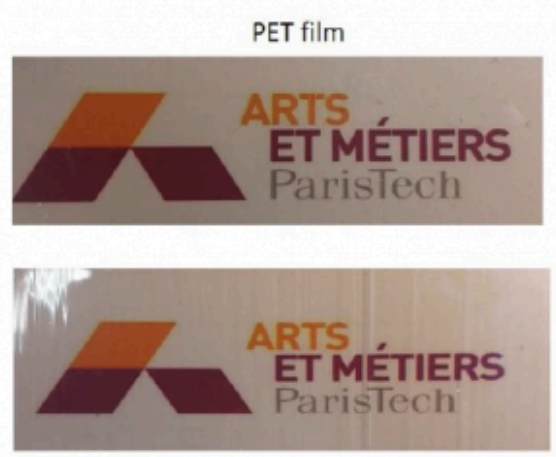

PET- PEF film

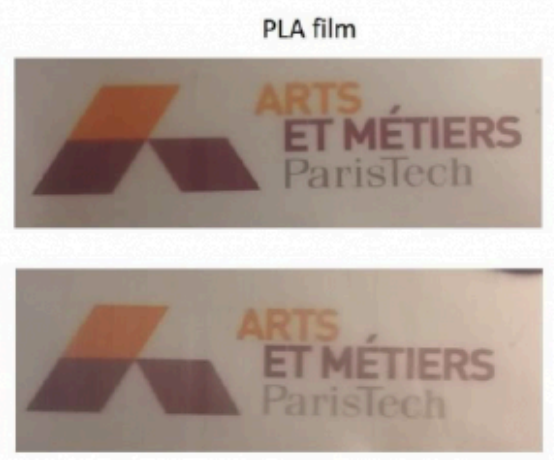

PLA - PEF film

Figure 1: film sample of PET, PLA, PET-PET and PLA-PEF

PET-PEF films presented a good transparency despite the high brownness of PEF slightly colouring the films. PLA-PEF films was slightly translucid and cream corresponding to the colour of PLA pellets due to the presence of additives.

If it was possible to fabricate all the 3, 128 and 512-layer PLA-PEF films, it was not possible to produce the 512-layer PET-PEF film due to a bad stretchability compared to PLA-PEF films.

The cross-sections of the 3-layer PET-PEF and PLA-PEF films exhibited a 3-layer structure with a PEF layer thickness in agreement with the expected values. The cross-section of the 128 and 512-layer PLA-PEF films revealed the presence of many layer break-ups [13] and typical blend morphologies with elongated droplets [14] were observed in both films. These layer break-ups occurring at such high thicknesses can be due to the presence of additives in PLA.

For the 128-layer PET-PEF films, continuous PEF layers were observed but with a wide thickness distribution, possibly due to high viscosity mismatch. 
a)

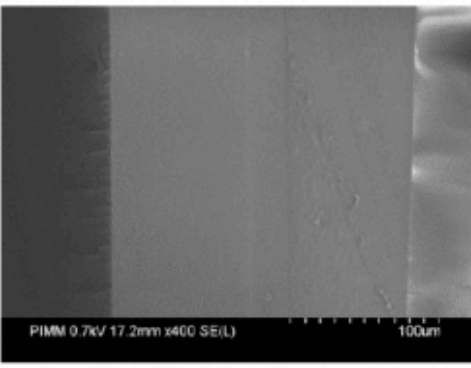

c)

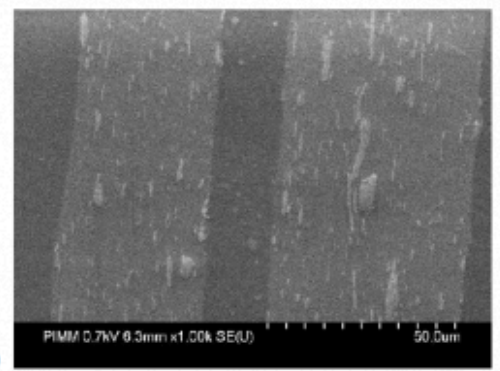

b)

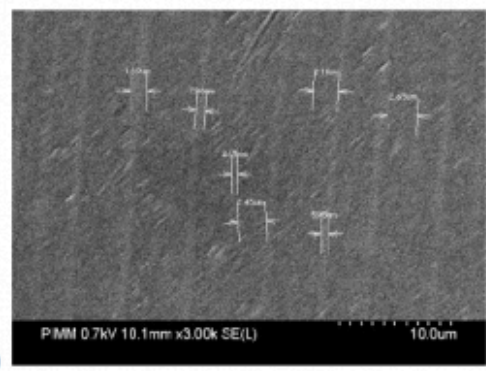

d)

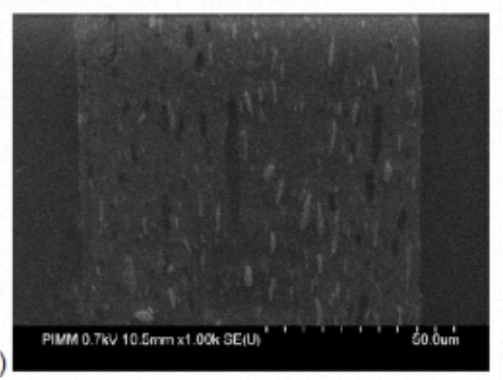

Fig. 2. Observation of the cross-section of a) 3-layer PET-PEF film, b) 128-layer PET-PEF film, c) 3-layer PLA-PEF film, d) 128-layer PLA-PEF film.

The oxygen gas barrier properties (OTR) of the films were measured at $23^{\circ} \mathrm{C}$ and $0 \% \mathrm{RH}$ (table 2 and 3). First, PLA, PET and PEF neat films were measured as reference (table 2). For PEF two different films were measured: extruded and thermopressed films fabricated from pellets. PLA exhibited classical results similar to those obtained for an INGEO amorphous PLA [15], while PET and PEF presented higher values compared to the literature [16]. Those values may be due to the very low crystallinity measured by DSC. Globally, we obtained the following expected results: OTR PLA $>>$ OTR PET > > OTR PEF.

Table 2. Values of PET, PLA and PEF crystallinity, Oxygen Transmission Rates (OTR) after the extrusion

\begin{tabular}{|l|c|c|}
\hline Film (and measured thickness in $\mu \mathrm{m})$ & $\begin{array}{c}\text { Crystallinity } \\
(\%)\end{array}$ & $\begin{array}{c}\text { OTR } \\
\left(\mathrm{cc} / \mathrm{m}^{2} \text {.day }\right)\end{array}$ \\
\hline PET $(100)$ & 5 & 38 \\
\hline PLA $(100)$ & 16 & 202 \\
\hline PEF $(65)$ & 1 & 6 \\
\hline PEF pellets as reference & 30 & - \\
\hline
\end{tabular}

Table 3 shows an improvement of a factor respectively 1.6 to 1.8 for 3-layer PET-PEF films and only 4 for PLA-PEF 3-layer film compared to PET and PLA respectively. The improvement was in agreement with the series permeability model for PET-PEF films, considering the amorphous state of PET and the medium crystallinity of PLA as measured by DSC, that did not affect strongly the PLA permeability [15]. But it was much lower than expected (factor 4 instead of 10) for PLA-PEF films. We do not have any explanation for this result at this stage since a PEF continuous layer and similar crystallinity degree were observed.

Table 3. Values of PEF layers thickness, crystallinity, Oxygen Transmission Rates (OTR) after the extrusion 
Relationship Between Crystallization, Mechanical and Gas Barrier Properties of Poly(eth...

\begin{tabular}{|l|c|c|c|c|}
\hline $\begin{array}{l}\text { Film } \\
\text { (and measured } \\
\text { thickness in } \mu \mathrm{m})\end{array}$ & $\begin{array}{c}\text { PEF } \\
\text { amount } \\
(\% \mathrm{w} / \mathrm{w})\end{array}$ & $\begin{array}{c}\text { PEF } \\
\text { nominal } \\
\text { thickness } \\
(\mathrm{nm})\end{array}$ & $\begin{array}{c}\text { PEF/PLA/PET } \\
\text { crystallinity(\%) }\end{array}$ & $\begin{array}{c}\text { OTR } \\
\left(\mathrm{cc} / \mathrm{m}^{2} \text {.day }\right)\end{array}$ \\
\hline $\begin{array}{l}\text { 3-layer } \\
\text { PET-PEF }(100)\end{array}$ & 10 & 10000 & $0 /-/ 6$ & 25 \\
\hline $\begin{array}{l}\text { 128-layer } \\
\text { PET-PEF }(100)\end{array}$ & 15 & 230 & $8 /-/ 8$ & 25 \\
\hline $\begin{array}{l}\text { 3-layer } \\
\text { PLA-PEF }(100)\end{array}$ & 10 & 10000 & $10 / 28 /-$ & 47 \\
\hline $\begin{array}{l}\text { 128-Layer } \\
\text { PLA-PEF }(100)\end{array}$ & 10 & 150 & $2 / 25 /-$ & 119 \\
\hline $\begin{array}{l}\text { 512-layer } \\
\text { PLA-PEF }(100)\end{array}$ & 10 & 60 & $5 / 17 /-$ & 128 \\
\hline
\end{tabular}

The 128 and 512-layer PLA-PEF films presented curiously higher OTR than the 3-layer ones, even though PEF crystallinity degrees decreased with 128 layers and 512 layers. It was certainly the consequence of the break-up of the PEF layers that could not act as a barrier layer for PLA. It can be explained a new time by the blend like morphology, where the barrier properties are finally not dependant of the crystallinity degree of the dispersed phase. Concerning PET-PEF films, 3-layer and 128-layer PET-PEF films presented finally the same OTR improvement independently of the PEF crystallinity degrees that were measured very different.

The classical tensile tests (Fig 3) were not discriminating, since they showed similar results for all the multilayers films, regardless of the test direction. No real modification of the mechanical properties has been observed when adding PEF and/or varying the number of layers for both PET-PEF and PLA-PEF films.
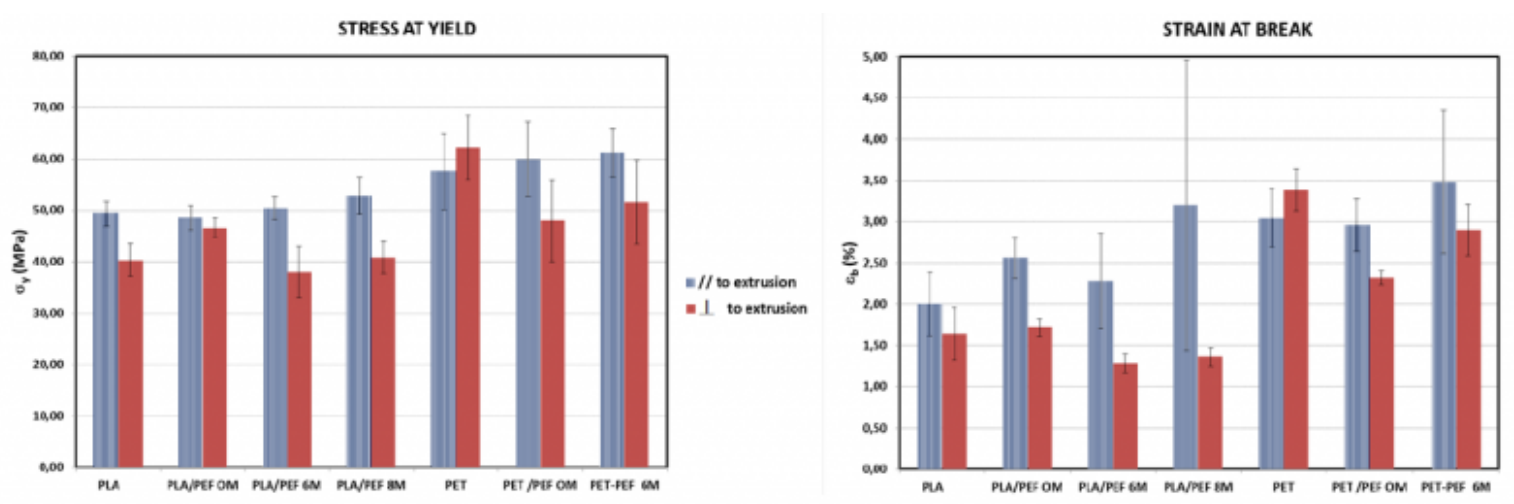

Fig. 3. Tensile tests: stress at yield and strain at break of the different films in the extrusion direction $(/ /)$ and perpendicular to extrusion $\left({ }^{\perp}\right)$

The puncture test measurements were actually much more discriminating. If no great variation of the puncture energy was obtained for PLA-PEF films, the layer multiplication clearly revealed its interest for PET-PEF films due to the high PEF brittleness. In fact, as shown in figure 4, adding PEF to PET in a 3-layer PET-PEF film led to a disastrous decrease of the puncture energy. By increasing the number of PEF layers, the high puncture energy of PET was almost recovered. This phenomenon may be due to the increase of the interfaces between the PET and the PEF layers that could limit the crack propagation. It was all the more interesting as PEF in 128-layer PET-PEF films had higher crystallinity that should usually lead to more brittleness. 


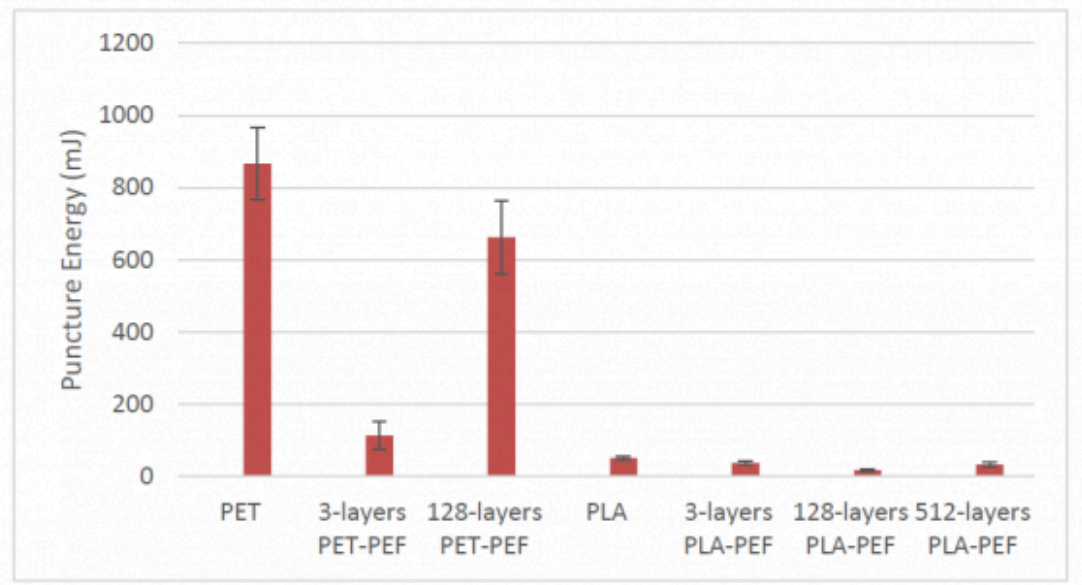

Fig. 4. Puncture test energy of the different PET-PEF and PLA-PEF films.

Post extrusion thermal annealing was performed on the films at a temperature of $140^{\circ} \mathrm{C}$ for 2 hours as described previously [18]. Annealing of the neat PLA and PEF films leaded to high increase of crystallinity and a decrease of a factor around 2 of their OTR (table 4). PEF became very brittle so that OTR could not be measured. The table 5 shows that PEF crystallinity degrees of the PET-PEF films increased, but to a lesser extent when PEF is confined. Even longer annealing time did not allow reaching the initial crystallinity degree measured on the as received PEF pellets (table 2). It was also observed that the increase of the PEF crystallinity led to a deterioration of the oxygen barrier properties. This surprising result, in contradiction with the results obtained on other PEF grade [17] and in the line of some results obtained on PET or PLA, requires more investigation before interpretation since PET crystallinity increased leading to wait for OTR decrease. PEF-PLA crystallinity were not been measured since the impact on the PEF crystallinity did not act directly on the gas barrier properties.

Table 4. Values of PET, PLA and PEF crystallinity, Oxygen Transmission Rates (OTR) after the extrusion and after the annealing stage

\begin{tabular}{|l|c|c|c|c|}
\hline & \multicolumn{2}{|c|}{ After extrusion } & \multicolumn{2}{c|}{ After annealing * } \\
\hline $\begin{array}{l}\text { Film (and } \\
\text { measured } \\
\text { thickness in } \\
\mu \mathrm{m})\end{array}$ & $\begin{array}{c}\text { Crystallinity } \\
(\%)\end{array}$ & OTR & $\begin{array}{c}\text { Crystallinity } \\
(\%)\end{array}$ & OTR \\
\hline PET (100) & & $\left(\mathrm{cc} / \mathrm{m}^{2}\right.$.day) \\
\hline PLA (100) & 5 & 38 & & 23 \\
\hline PEF (65) & 16 & 202 & 26 & 81 \\
\hline
\end{tabular}


Relationship Between Crystallization, Mechanical and Gas Barrier Properties of Poly(eth...

\begin{tabular}{|c|c|c|c|c|}
\hline & \multicolumn{2}{|c|}{ After extrusion } & \multicolumn{2}{|c|}{ After annealing } \\
\hline $\begin{array}{l}\text { Film (and } \\
\text { measured } \\
\text { thickness in } \\
\mu \mathrm{m})\end{array}$ & $\begin{array}{c}\text { PEF/PLA/PET } \\
\text { crystallinity } \\
(\%)\end{array}$ & $\begin{array}{c}\text { OTR } \\
\text { (cc/m².day) }\end{array}$ & $\begin{array}{c}\text { PEF/PLA/PET } \\
\text { crystallinity } \\
(\%)\end{array}$ & $\begin{array}{r}\text { OTR } \\
\text { (cc/m } \mathrm{m}^{2} \text {.day) }\end{array}$ \\
\hline $\begin{array}{l}\text { 3-layer } \\
\text { PET-PEF } \\
(100)\end{array}$ & $0 /-/ 6$ & 25 & $10 /-/ 27$ & 40 \\
\hline $\begin{array}{l}\text { 128-layer } \\
\text { PET-PEF } \\
(100)\end{array}$ & $8 /-/ 8$ & 25 & $14 /-/ 25$ & 30 \\
\hline
\end{tabular}

Table 5. Values of the PEF, PLA and PET crystallinity and Oxygen Transmission Rates (OTR) of the mutilayered films after the extrusion and after the annealing stage

\begin{tabular}{|l|c|c|c|c|}
\hline $\begin{array}{l}\text { 3-layer } \\
\text { PLA-PEF } \\
(100)\end{array}$ & $10 / 28 /-$ & 47 & n.d & 48 \\
\hline $\begin{array}{l}\text { 128-Layer } \\
\text { PLA-PEF } \\
(100)\end{array}$ & $2 / 25 /-$ & 119 & n.d & n.d \\
\hline $\begin{array}{l}512-\text { layer } \\
\text { PLA-PEF } \\
(100)\end{array}$ & $5 / 17 /-$ & 128 & n.d & 158 \\
\hline
\end{tabular}

\section{Conclusion}

This work showed the possibility of coextruding 10 to 15\%wt. of PEF with PLA or PET. $100 \mu \mathrm{m}$ thick PLA-PEF and PET-PEF multilayer films with various layer number/thicknesses were processed and presented different features. The multilayer PET-PEF films were transparent while the PLA-PEF films were rather translucid, probably due to the PLA formulation. The layers were continuous at the nanoscale for PET-PEF films, but layer breakups occurred in the 128 or 512-layer PLA-PEF films exhibiting blend morphologies. The 3-layer PET-PEF films followed the series law for oxygen permeability measurements while it was not the case for the 3-layer PLA-PEF films. It was related to crystallinity degrees; PEF was completely amorphous in PET-PEF films while it was a little more crystallized in PLA-PEF films. Concerning the 128 and 512-layer PLA-PEF films it was highlighted that the blend structure did not improve logically the oxygen barrier properties compared to PLA. It was the same for the mechanical properties. Concerning the PET-PEF films, the 128-layer film presented the same oxygen permeability than this one of the 3-layer one despite a higher PEF crystallinity.

A highly positive effect of the layer multiplication was revealed thanks to the puncture tests. It was also highlighted that puncture test measurements were disastrous for 3-layer PET-PEF films certainly due to the high brittleness of the PEF despite its amorphous state. The layer multiplication permitted to recover the PET puncture test measurement by introducing PEF. Crystallinity was correlated to those behaviours especially for PET-PEF films. PEF crystallinity increase due to layer multiplication did not seem to act classically on both gas barrier and mechanical properties. Compared to PEF classical behaviour indicated in different works [8], increasing our PEF crystallinity leaded to lower gas barrier properties and to improve the puncture properties.

This result was confirmed by the film annealing. Annealing step on PET-PEF and PLA-PEF films did not lead also to an improvement of the gas barrier properties despite the crystallinity increase, it was even the inverse. This work showed 
clearly the unusual relationship between crystallinity and gas barrier and mechanical properties of the PEF of our study certainly linked to a confinement effect.

\section{Acknowledgements}

The authors want to thank the European Commission for the funding of the H2020 Mypack Project.

\section{Bibliography}

[1] Auras, R, Harte, B, Selke, S, An Overview of Polylactides as Packaging Materials, Macromol. Biosci. 2004, 4, 835 -864

[2] Guinault, A., Sollogoub, C., Ducruet, V., \& Domenek, S. (2012). Impact of crystallinity of poly (lactide) on helium and oxygen barrier properties. European Polymer Journal, 48(4), 779-788.

[3] Fernandes Nassar, S., Delpouve, N., Sollogoub, C., Guinault, A., Stoclet, G., Régnier, G., \& Domenek, S. (2020). Impact of nanoconfinement on polylactide crystallization and gas barrier properties. ACS applied materials \& interfaces, 12(8), 9953-9965.

[4] Burgess, S. K., Karvan, O., Johnson, J. R., Kriegel, R. M., \& Koros, W. J. (2014). Oxygen sorption and transport in amorphous poly (ethylene furanoate). Polymer, 55(18), 4748-4756.

[5] Wang, G., Jiang, M., Zhang, Q., Wang, R., Zhou, G., (2017) Biobased copolyesters: synthesis, crystallization behavior, thermal and mechanical properties of poly(ethylene glycol sebacate-co-ethylene glycol 2,5-furan dicarboxylate), RSC Adv., 7, 13798-13807

[6] Konstantopoulou, M., Terzopoulou, Z., Nerantzaki, M., Tsagkalias, J., Achilias D.S., Bikiaris D.N., Exarhopoulos, S., Papageorgiou, D.G., Papageorgiou, G.Z. (2017), Poly(ethylene furanoate-co-ethylene terephthalate) biobased copolymers: Synthesis, thermal properties and cocrystallization behavior, European Polymer Journal, 89, 349-366.

[7] Poulopoulou, N., Smyrnioti, D., Nikolaidis, G.N., Tsitsimaka, I., Christodoulou, E., Bikiaris, D.N., Charitopoulou, M.A., Achilias, D.S., Kapnisti, M., Papageorgiou, G.Z. (2020), Sustainable Plastics from Biomass: Blends of Polyesters Based on 2,5-Furandicarboxylic Acid. Polymers 12, 225.

[8] Paszkiewicz, S., Irska, I., Piesowicz, E. (2020), Environmentally Friendly Polymer Blends Based on Post-Consumer Glycol-Modified Poly(Ethylene Terephthalate) (PET-G) Foils and Poly(Ethylene 2,5-Furanoate) (PEF): Preparation and Characterization. Materials 13, 2673.

[9] Wang, H., Keum, J. K., Hiltner, A., \& Baer, E. (2009). Confined crystallization of PEO in nanolayered films impacting structure and oxygen permeability. Macromolecules, 42(18), 7055-7066.

[10] Messin, T., Marais, S., Follain, N., Guinault, A., Gaucher, V., Delpouve, N., Sollogoub, C. (2020), Biodegradable PLA/ PBS multinanolayer membrane with enhanced barrier performances, Journal of Membrane Science, 598, 117777

[11] Bironeau, A., Dirrenberger, J., Sollogoub, C., MIQUELARD-GARNIER, G., \& Roland, S. (2016). Evaluation of morphological representative sample sizes for nanolayered polymer blends. Journal of microscopy, 264(1), 48-58.

[12] Kazaryan L. G., Medvedeva, F.M. (1968) X-ray study of poly (ethylene furan-2, 5-dicarboxylate) structure, Vysokomol. Soedin., Ser. B.,10, 305.

[13] Bironeau, A., Salez, T., Miquelard-Garnier, G., \& Sollogoub, C. (2017). Existence of a critical layer thickness in PS/ PMMA nanolayered films. Macromolecules, 50(10), 4064-4073. 
Relationship Between Crystallization, Mechanical and Gas Barrier Properties of Poly(eth...

[14] Feng, J., Zhang, Z., Bironeau, A., Guinault, A., Miquelard-Garnier, G., Sollogoub, C., Olah, A. \& Baer, E. (2018). Breakup behavior of nanolayers in polymeric multilayer systems-Creation of nanosheets and nanodroplets. Polymer, 143, 19-27.

[15] Fernandes Nassar, S., Guinault, A., Delpouve, N., Divry, V., Ducruet, V., Sollogoub, C., \& Domenek, S. (2017). Multiscale analysis of the impact of polylactide morphology on gas barrier properties. Polymer (United Kingdom), 108, $163-172$

[16] Burgess, S, K, Leisen, J,E, Kraftschik, B,E, Mubarak, C,R, Kriegel, R,M, Koros, W,J, (2014). Chain Mobility, Thermal, and Mechanical Properties of Poly(ethylenefuranoate) Compared to Poly(ethylene terephthalate). Macromolecules, 47, $1383-1391$

[17] Burgess, S,K, Wenz, G,B, Kriegel, R,M, Koros, W,J. (2016). Penetrant transport in semicrystalline poly(ethylene furanoate). Polymer, 98, 305-310

[18] Codou, A, Guigo N. Van Berkel J, De Jong E, Sbirrazzuoli, N, (2014) Non-isothermal Crystallization Kinetics of Biobased Poly(ethylene 2,5-furandicarboxylate) Synthesized via the Direct Esterification Process, Macromol. Chem. Phys.215, 2065-2074

PDF automatically generated on 2021-05-21 12:48:17

Article url: https://popups.uliege.be/esaform21/index.php?id=1589

published by ULiège Library in Open Access under the terms and conditions of the CC-BY License (https://creativecommons.org/licenses/by/4.0) 